\title{
Event plane resolution correction for azimuthal anisotropy in wide centrality bins
}

\author{
Hiroshi Masui, Alexander Schmah, A.M. Poskanzer \\ Lawrence Berkeley National Laboratory, Berkeley, CA 94720, USA
}

\begin{abstract}
We provide a method to correct the observed azimuthal anisotropy in heavy-ion collisions for the event plane resolution in a wide centrality bin. This new procedure is especially useful for rare particles, such as $\Omega$ baryons and $J / \psi$ mesons, which are difficult to measure in small intervals of centrality. Based on a Monte Carlo calculation with simulated $v_{2}$ and multiplicity, we show that some of the commonly used methods have a bias of up to $15 \%$.
\end{abstract}

Keywords: Azimuthal anisotropy, flow, event plane resolution

\section{Introduction}

Azimuthal anisotropy is one of the key observables to study the properties of matter created in high energy heavy-ion collisions (see e.g. [1]). It is characterized by the Fourier decomposition of the azimuthal particle distribution with respect to the participant plane [2]

$$
\frac{d N}{d \phi}=\frac{N_{0}}{2 \pi}\left(1+2 \sum_{n=1}^{\infty} v_{n} \cos \left[n\left(\phi-\Psi_{\mathrm{PP}_{\mathrm{n}}}\right)\right]\right),
$$

where $N_{0}$ is the number of particles in the event, $v_{n}$ is the $n$-th harmonic coefficient, $\phi$ is the azimuthal angle of particles and $\Psi_{\mathrm{PP}_{n}}$ is the azimuthal angle of the $n^{\text {th }}$ harmonic participant plane. The participant plane is the symmary plane of the participants.

One of the standard methods is to extract $v_{n}$ by using the reconstructed event plane from the detected participant particles [3]. The most important task in this method is to estimate the participant plane from the measured particles for each harmonic $n$. The estimated participant plane is defined as the event plane $\Psi_{n}\left(-\pi / n \leq \Psi_{n}<\pi / n\right)$, but due to the finite multiplicity in nuclear collisions and fluctuations, the event plane can be different from the participant plane. The observed $v_{n}^{\text {obs }}(M)$ for a given, small, centrality range $M$ must be corrected by the event plane resolution $\mathcal{R}_{n}(M)$ in order to take into account the difference between true participant plane and event plane

Email addresses: hirmasui@gmail . com (Hiroshi Masui), ASchmah@lbl.gov (Alexander Schmah), AMPoskanzer@lbl.gov (A.M. Poskanzer)

$$
v_{n}(M)=\frac{\left\langle\left\langle\cos \left[n\left(\phi-\Psi_{m}\right)\right]\right\rangle\right\rangle_{M}}{\left\langle\cos \left[n\left(\Psi_{m}-\Psi_{\mathrm{PP}_{n}}\right)\right]\right\rangle_{M}} \equiv \frac{v_{n}^{\mathrm{obs}}(M)}{\mathcal{R}_{n}(M)},
$$

where $m$ is the harmonic of the event plane and $n=k m$ is the harmonic of interest. The integer $k$ is taken as unity in this paper. Brackets denote the average over events, while double brackets denote the average over particles in all events. Subscript $M$ on the bracket emphasizes that the average is taken for a given centrality $M$. For simplicity, we will omit $M$ from the observables, for example, we will write $N_{0}$ instead of $N_{0}(M)$. In experiments the participant plane angle $\Psi_{\mathrm{PP}_{n}}$ in Eq. (1) is not known. Therefore at least two subevent planes are necessary in order to calculate the event plane resolution [3]. An average $v_{n}$ over a wider centrality range $R$ can be calculated once $v_{n}^{\text {obs }}$ and $\mathcal{R}_{n}$ are determined within the range $R$

$$
\frac{\int_{R} d M N_{0} \frac{v_{n}^{\mathrm{obs}}}{\mathcal{R}_{n}}}{\int_{R} d M N_{0}} \equiv\left\langle\frac{v_{n}^{\mathrm{obs}}}{\mathcal{R}_{n}}\right\rangle=\left\langle v_{n}\right\rangle .
$$

We introduced brackets $\langle\ldots\rangle$ for simplicity, which denote the average over a wide centrality range weighted by particle multiplicity $N_{0}$ for a given phase space (e.g. for a given transverse momentum range).

$\mathcal{R}_{n}$ depends on multiplicity and $v_{n}$ itself, therefore Eq. (3) requires that $v_{n}^{\text {obs }}$ and $\mathcal{R}_{n}$ are measured in sufficiently small centrality intervals. However, for rare particles (e.g. $\Omega, J / \psi$ ) it is not always possible to do so. One of the conventional approaches (e.g. see Ref. [4]) 
is to average $v_{n}^{\text {obs }}$ as well as $\mathcal{R}_{n}$ separately in a wide 90 centrality range, weighted by the corresponding particle yields, and then divide $\left\langle v_{n}^{\text {obs }}\right\rangle$ by $\left\langle\mathcal{R}_{n}\right\rangle$. However, this approach systematically overestimates $v_{n}$ as we will discuss in Section 3. The main point of this paper is the following inequality

$$
\left\langle\frac{v_{n}^{\mathrm{obs}}}{\mathcal{R}_{n}}\right\rangle \neq \frac{\left\langle v_{n}^{\mathrm{obs}}\right\rangle}{\left\langle\mathcal{R}_{n}\right\rangle} \neq\left\langle v_{n}^{\mathrm{obs}}\right\rangle\left\langle\frac{1}{\mathcal{R}_{n}}\right\rangle
$$

where the left hand side is the correct average over a wide centrality bin, while the right hand side shows commonly used approximations. However, it is easy to avoid these approximations.

We will show the proper way to correct for the finite event plane resolution in wide centrality bins. Eventhough the resolution is an event-by-event quantity, the calculation of the sum over individual particles for the flow coefficients must be done with the inverse of the reolution for the proper centrality. In Section 2, we derive the equations used to calculate $\left\langle v_{n}\right\rangle$ in our approach. We also show that the derived equations are equivalent to the average calculated from narrow centrality bins (see Eq. (3)). In Section 3, we show a simple Monte Carlo simulation to demonstrate the validity of the method, for the case of $v_{2}$. Based on a preliminary version of this paper [5], the method has been appled allready in several publications $[6,7,8]$.

\section{Implementation}

We now show two practical implementations to cor- 105 rect $v_{n}$ for the event plane resolution in wide centrality 106 bins. There are two or three steps to calculate $v_{n}$ in wide ${ }^{107}$ centrality bins

1. Determine the event plane resolution $\mathcal{R}_{n}$ as a function of $M$ in narrow centrality ranges.

2. Analyse $v_{n}$ with the weights $1 / \mathcal{R}_{n}$ for any, wide, 11 centrality range of interest.

3. In addition for the event-plane method, one must ${ }^{113}$ multiply the result by the average weight.

In the following subsections, we discuss detailed im- 116 plementations of how to apply corrections for different types of particle identification. For the sake of sim- 117 plicity, we assume that non-flow effects are negligible, 118 and all correlations between particles are induced by 119 flow. A systematic study of other effects can be found 120 in Ref. [8].
The azimuthal particle distribution with respect to the event plane can be written as

$$
\frac{d N}{d\left(\phi-\Psi_{m}\right)}=\frac{N_{0}}{2 \pi}\left(1+2 \sum_{n}^{\infty} v_{n}^{\mathrm{obs}} \cos \left[n\left(\phi-\Psi_{m}\right)\right]\right) \text {. }
$$

\section{2.1. Event-by-event particle identification}

If the particle of interest can be identified on an event-by-event basis, one can directly calculate $\cos \left[n\left(\phi-\Psi_{m}\right)\right]$ for every particle, corrected with the event plane resolution for the corresponding centrality $M$

$$
\frac{\cos \left[n\left(\phi-\Psi_{m}\right)\right]}{\mathcal{R}_{n}},
$$

where $\mathcal{R}_{n}(M)$ is supposed to be averaged over many events in advance. The event and centrality average in the range of multiplicities $R$ of term (6) over $\phi-\Psi_{m}$ reduces in this case to Eq. (3):

$$
\begin{aligned}
& \frac{\int_{R} d M \int_{0}^{2 \pi} d\left(\phi-\Psi_{m}\right) \frac{d N}{d\left(\phi-\Psi_{m}\right)} \frac{\cos \left[n\left(\phi-\Psi_{m}\right)\right]}{\mathcal{R}_{n}}}{\int_{R} d M \int_{0}^{2 \pi} d\left(\phi-\Psi_{m}\right) \frac{d N}{d\left(\phi-\Psi_{m}\right)}} \\
& =\frac{\int_{R} d M N_{0} \frac{v_{n}^{\text {obs }}}{\mathcal{R}_{n}}}{\int_{R} d M N_{0}}=\left\langle v_{n}\right\rangle
\end{aligned}
$$

\subsection{Statistical particle identification}

There are two approaches in case the particle yield of interest can only be extracted statistically: the invariant mass fit method and the event plane method. The invariant mass fit method is almost equivalent to the one introduced in Section 2.1, while the event plane method needs one additional step to obtain the final $v_{n}$.

\subsubsection{Invariant mass fit method}

The invariant mass method [9] is quite useful to analyse particles that are detected through their decay products, such as $K_{s}^{0} \rightarrow \pi^{+} \pi^{-}, \Lambda \rightarrow p \pi^{-}$and so on. The point of this method is to calculate the mean azimuthal 
angle relative to the event plane, $\cos \left[n\left(\phi-\Psi_{m}\right)\right]$, as a ${ }_{158}$ function of invariant mass $M_{\text {inv }}$

$$
\begin{aligned}
v_{n}^{S+B}\left(M_{\mathrm{inv}}\right) & =\left\langle\cos \left[n\left(\phi-\Psi_{m}\right)\right]_{\mathrm{inv}}\right\rangle, \\
& =v_{n}^{S} \frac{S}{S+B}\left(M_{\mathrm{inv}}\right)+v_{n}^{B}\left(M_{\mathrm{inv}}\right) \frac{B}{S+B}\left(M_{\mathrm{inv}}\right) .
\end{aligned}
$$

where $S$ is the signal yield peaked at the mass of the 159 partcle, $B$ is the smooth background yield, $v_{n}^{S}, v_{n}^{B}$ and 160 $v_{n}^{S+B}$ are the $v_{n}$ for signal, background and total parti- 161 cles, respectively. Signal and background contributions 162 are decomposed by taking into account the measured ${ }_{163}$ signal-to-background ratio and using a parametrization of the background $v_{n}^{B}$ shape by assuming that $B\left(M_{\mathrm{inv}}\right)$, and $v_{n}^{B}\left(M_{\mathrm{inv}}\right) B\left(M_{\mathrm{inv}}\right)$ are smooth functions of $M_{\mathrm{inv}}$ [9], while $v_{n}^{S} S\left(M_{\text {inv }}\right)$ is peaked at $M_{\text {inv }}$. Since the average cosine is calculated in this approach, one can directly extract the $v_{n}^{S}$ by subtracting the background contribution. The only modification is to add a weight $1 / \mathcal{R}_{n}$ on an event-by-event basis when one fills the histograms for $\cos \left[n\left(\phi-\Psi_{m}\right)\right]$ versus invariant mass similar to Eq. (7).

\subsubsection{Event plane method}

The event plane method [3] with $\phi-\Psi_{m}$ binning is an alternative approach to a $v_{n}$ analysis when particle yields can be determined only statistically after a background is subtracted. This method requires the decomposition of signal and background in several $\phi-\Psi_{m}$ bins (typically 10-20), while the invariant mass fit method only requires the decomposition once. The event plane method does not need the assumption of a background $v_{n}$ shape. Therefore this method has some advantage, especially when the signal to background ratio is poor.

The first step in the event plane method is the signal extraction for a given $\phi-\Psi_{m}$ bin

$$
N^{\mathcal{R}}\left(\phi-\Psi_{m}\right)=\int_{R} d M \frac{1}{\mathcal{R}_{n}} \frac{d N}{d\left(\phi-\Psi_{m}\right)},
$$

where $N^{\mathcal{R}}\left(\phi-\Psi_{m}\right)$ is the number of particles for a given $\phi-\Psi_{m}$ bin, weighted for each centrality bin with the inverse of the event plane resolution. The difference to the conventional method is the weight $1 / \mathcal{R}_{n}$ on the particle yields which will properly take into account the centrality dependence of the event plane resolution. Second, one integrates over $\phi-\Psi_{m}$ to calculate $v_{n}$

$$
\frac{\int_{0}^{2 \pi} d\left(\phi-\Psi_{m}\right) N^{\mathcal{R}}\left(\phi-\Psi_{m}\right) \cos \left[n\left(\phi-\Psi_{m}\right)\right]}{\int_{0}^{2 \pi} d\left(\phi-\Psi_{m}\right) N^{\mathcal{R}}\left(\phi-\Psi_{m}\right)}
$$

and then using Eq. (9) gets

$\left\langle v_{n}^{\mathcal{R}}\right\rangle=$

$$
\frac{\int_{R} d M \int_{0}^{2 \pi} d\left(\phi-\Psi_{m}\right) \frac{d N}{d\left(\phi-\Psi_{m}\right)} \frac{\cos \left[n\left(\phi-\Psi_{m}\right)\right]}{\mathcal{R}_{n}}}{\int_{R} d M \int_{0}^{2 \pi} d\left(\phi-\Psi_{m}\right) \frac{d N}{d\left(\phi-\Psi_{m}\right)} \frac{1}{\mathcal{R}_{n}}} .
$$

Note that this equation includes the $1 / \mathcal{R}_{n}$ weights on the particle yields in the denominator. One could immediately notice that the numerator is identical to Eq. (7) with the additional normalization $1 /\left(\int_{R} d M N_{0}\right)$. The denominator becomes

$$
\int_{R} d M \frac{N_{0}}{\mathcal{R}_{n}}=\left(\int_{R} d M N_{0}\right)\left\langle\frac{1}{\mathcal{R}_{n}}\right\rangle .
$$

The normalization factor $\int_{R} d M N_{0}$ is cancelled out between numerator and denominator. Thus $\left\langle v_{n}\right\rangle$ is obtained from Eq. (10) by multiplying by the average weight, $\left\langle 1 / \mathcal{R}_{n}\right\rangle$ :

$$
\left\langle v_{n}\right\rangle=\left\langle v_{n}^{\mathcal{R}}\right\rangle\left\langle\frac{1}{\mathcal{R}_{n}}\right\rangle .
$$

We would like to emphasize two important points for the event plane method. First, one must take an average of the inverse event plane resolution $1 / \mathcal{R}_{n}$ (not an average of $\mathcal{R}_{n}$ ). Second, the integral $\int_{R} d M$ in Eq. (10) for both numerator and denominator should be calculated in the same phase space. For instance, if one measures the $v_{n}$ as a function of transverse momentum $p_{T}$, one should calculate $\left\langle 1 / \mathcal{R}_{n}\right\rangle$ for each $p_{T}$ bin.

This new implementation can also be applied for the scalar product method $[10,11]$. The scalar product method is an extension of the methods described in this paper where in addition to the event plane angle the magnitude of the Q-vector [3] is considered for both $v_{n}^{\text {obs }}$ and $\mathcal{R}_{n}$.

\section{Simulation results}

In this section we validate our implementation by a simple Monte Carlo simulation for $v_{2}\left(p_{T}\right)$. As we have mentioned earlier, this approach can be applied for any harmonic of interest.

Figure 1 shows the input multiplicity distribution in $0-80 \%$ centrality for the 50 million generated events. The event centrality classes are determined by using the multiplicity distribution as it is typically done in the experimental data. We divide the multiplicity distribution into 16 bins with $5 \%$ increments within $0-80 \%$. 


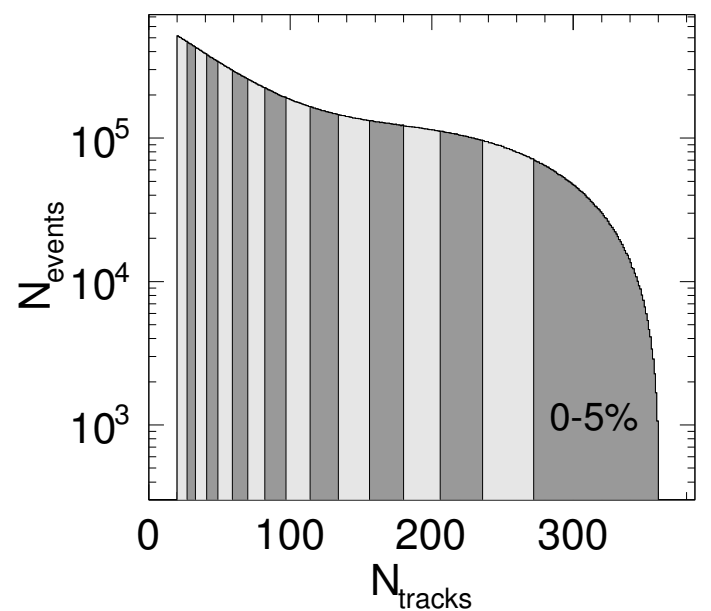

Figure 1: Input multiplicity distribution in the simple Monte Carlo simulation. The shaded area show $0-5 \%, 5-10 \%, \ldots, 75-80 \%$ centrality classes.

For every event a number of tracks according to the input multiplicity distribution was sampled. A Boltzmann like $p_{T}$ distribution from $0.25-3 \mathrm{GeV} / c$ was used. The input $v_{2}^{\text {in }}\left(p_{T}\right)$ for every centrality bin was fixed to an empirical parametrization of the following form

$$
v_{2}^{\text {in }}\left(p_{T}\right)=A\left(1-e^{-p_{T}}\right)\left(\frac{a}{1+e^{-\left(p_{T}-b\right) / c}}-d\right)
$$

which describes the shape of observed $v_{2}\left(p_{T}\right)$ in heavy ion collisions. The parameter $A$ was increased towards more peripheral centralities. Furthermore we have added for every event a Gaussian smearing on $v_{2}^{\text {in }}$ with a width of 0.05 . Figure 2 depicts the used $v_{2}^{\text {in }}\left(p_{T}\right)$ for the 16 centrality bins. The event Q-vector was calculated according to Ref. [3].

Figure 3 shows the random subevent plane resolution as a function of centrality. For each event, particles are randomly divided into two different groups in order to evaluate the resolution $\mathcal{R}_{2}$. The result shown here is the subevent plane resolution by using Eq. (14) in Ref. [3]. The resolution reaches a maximum of 0.45 around $30 \%$ centrality, it decreases towards more central and peripheral events because of a lower $v_{2}$ in central and less multiplicity in peripheral events. Then the full event plane resolution, which is used in this paper, is calculated from Eq. (11) in Ref. [3].

Figure 4 shows an example of a particle azimuthal distribution with respect to the second harmonic event plane in a narrow $p_{T}$ bin. The particle yields are weighted by the inverse event plane resolution as indi-

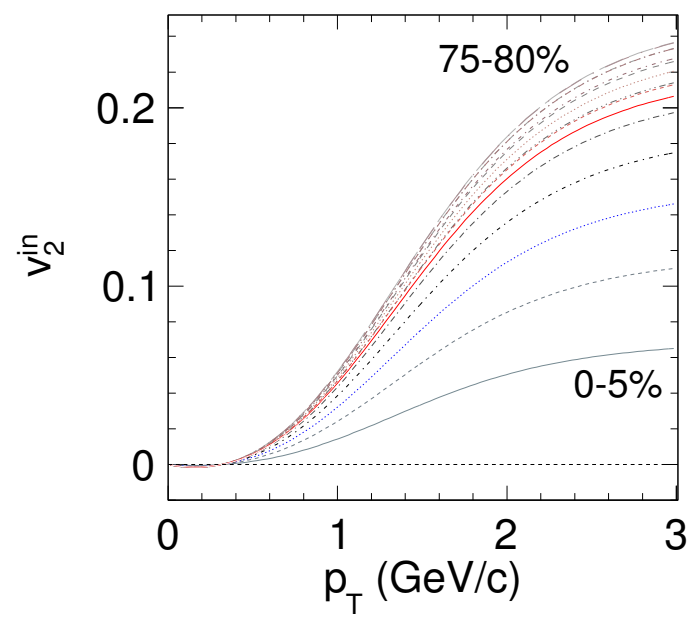

Figure 2: (Color online) Input $v_{2}\left(p_{T}\right)$ distributions for different centralities. Each line corresponds to a $5 \%$ centrality range. $v_{2}^{\text {in }}\left(p_{T}\right)$ increases towards more peripheral events. The applied Gaussian smearing is not shown.

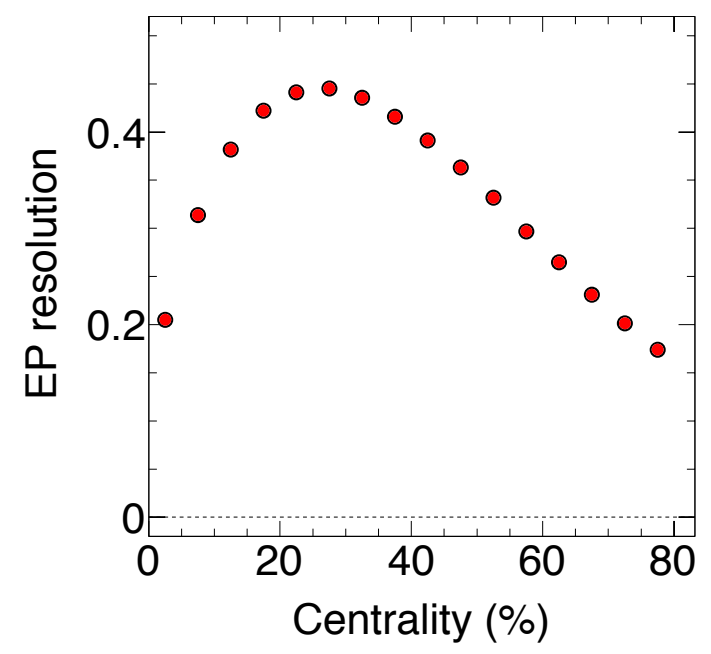

Figure 3: (Color online) Subevent-plane resolution for the second harmonic as a function of centrality. The resolution is calculated from random subevents. 


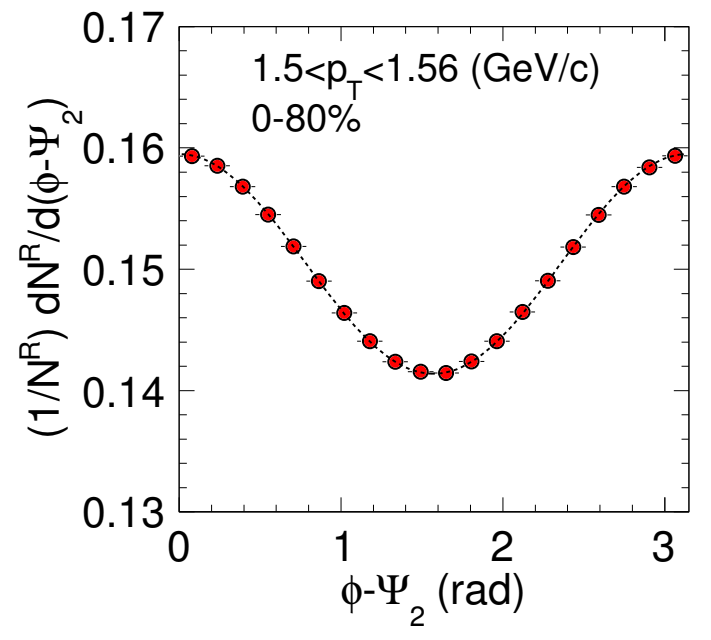

Figure 4: (Color online) Azimuthal distribution of particles with respect to the second harmonic event plane at $1.5<p_{T}<1.56 \mathrm{GeV} / c$ in $0-80 \%$ centrality bin. The dashed line is the fit result for $1+2 v_{2}^{\mathrm{obs}} \cos \left[2 \phi-2 \Psi_{2}\right]$ cated by $N^{\mathcal{R}}$ in the y-axis title. The dashed line represents a fit with $1+2 v_{2}^{\mathrm{obs}, \mathcal{R}} \cos \left[2 \phi-2 \Psi_{2}\right]$. The yield extraction and fit are repeated for all other $p_{T}$ bins.

Figure 5 shows $\left\langle v_{2}\right\rangle$ as a function of $p_{T}$ in the 0$80 \%$ centrality bin. For comparison, we also plot the observed $\left\langle v_{2}^{\mathrm{obs}}\right\rangle$ without resolution correction as shown by solid grey circles. The difference between observed and corrected $\left\langle v_{2}\right\rangle$ gives the size of the resolution correction. The input $\left\langle v_{2}^{\text {in }}\right\rangle$ of the simulation is shown as a magenta solid line. We tested both, the direct cosine calculation from Eq. (7) and the event plane method from Eq. (11), as shown by open blue stars and solid blue stars, respectively in panel (a) and (b). One can see that both direct cosine and event plane methods are consistent with the input $\left\langle v_{2}^{\text {in }}\right\rangle$ within $0.3 \%$. Panel (b) shows the observed $\left\langle v_{2}^{\text {obs }}\right\rangle$, corrected by the inverse mean event plane resolution $\langle\mathcal{R}\rangle^{-1}$ (green line) and the mean inverse event plane resolution $\left\langle\mathcal{R}^{-1}\right\rangle$ (red line). The relative difference $\Delta\left\langle v_{2}\right\rangle$ of the corrected $\left\langle v_{2}\right\rangle$ values to the input $\left\langle v_{2}^{\text {in }}\right\rangle$ is shown in the lower panels. For the correction with $\langle\mathcal{R}\rangle^{-1}$ and $\left\langle\mathcal{R}^{-1}\right\rangle$ we get a $\Delta\left\langle v_{2}\right\rangle /\left\langle v_{2}^{\text {in }}\right\rangle$ of $7 \%$ and $15 \%$, respectively. If there are enough data the resolution correction should be done separately for each $p_{T}$ bin.
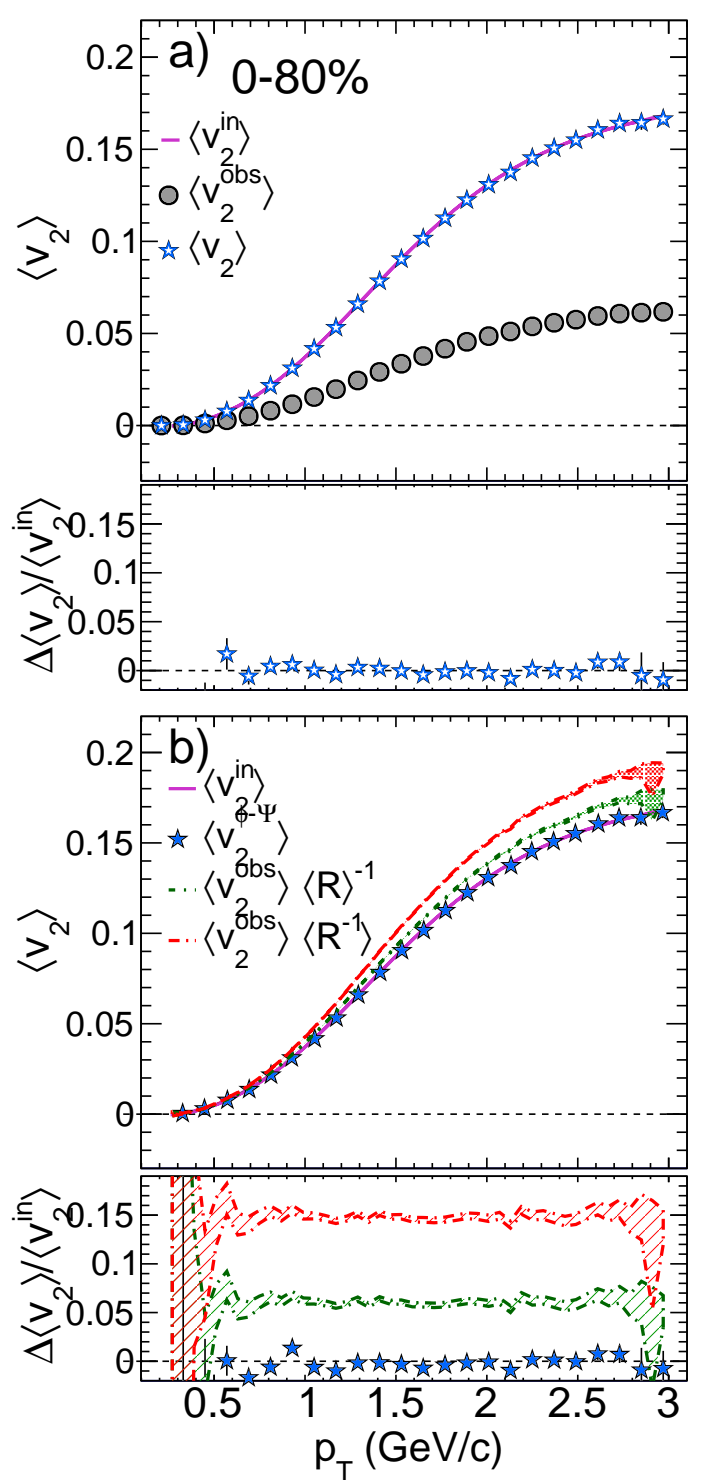

Figure 5: (Color online) Results of $v_{2}$ as a function of $p_{T}$ with different approaches of resolution corrections. The magenta solid line corresponds to the input $\left\langle v_{2}\right\rangle$, gray filled circles are the observed $\left\langle v_{2}^{\text {obs }}\right\rangle$ without resolution correction, blue open stars in panel (a) are from the direct cosine calculation from Eq. (7), blue filled stars in panel (b) are from the event plane method from Eq. (11). Other lines show the observed $\left\langle v_{2}^{\text {obs }}\right\rangle$ with different approximate mean event plane resolution corrections. 


\section{Conclusions}

We have introduced an implementation to avoid event plane resolution effects of wide centrality bins on $v_{n}$ measurements. In this new approach an inverse event plane resolution weight as a function of multiplicity is added on an event-by-event basis to the cosine term or to the particle yields. We confirmed that our approach reproduces the input $\left\langle v_{2}^{\text {in }}\right\rangle\left(p_{T}\right)$ in a wide centrality bin, whereas the conventional correction using the mean resolution overestimates the elliptic flow coefficient by $7 \%$ for minimum bias collisions. Surprisingly, usng the mean inverse resolution, which was thought to be a better approximatiion, overestimates the result by twice as much.

\section{Acknowledgements}

We thank Jean-Yves Ollitrault, Shusu Shi, and Xu Sun for discussions. This paper was funded in part by the US Department of Energy under Contract No. DEAC03-76SF00098.

[1] S. A. Voloshin, A. M. Poskanzer and R. Snellings, "Collective phenomena in non-central nuclear collisions," arXiv:0809.2949 [nucl-ex].

[2] S. Voloshin and Y. Zhang, "Flow study in relativistic nuclear collisions by Fourier expansion of Azimuthal particle distributions," Z. Phys. C 70, 665 (1996) doi:10.1007/s002880050141 [hep-ph/9407282].

[3] A. M. Poskanzer and S. A. Voloshin, "Methods for analyzing anisotropic flow in relativistic nuclear Phys. Rev. C 58, 1671 (1998) [nucl-ex/9805001].

[4] M. M. Aggarwal et al. [STAR Collaboration], " $K^{* 0}$ production in $\mathrm{Cu}+\mathrm{Cu}$ and $\mathrm{Au}+\mathrm{Au}$ collisions at $\sqrt{s_{N} N}=62.4 \mathrm{GeV}$ and 200 GeV,’ Phys. Rev. C 84, 034909 (2011) [arXiv:1006.1961 [nuclex]].

[5] H. Masui and A. Schmah, "Event plane resolution correction for azimuthal anisotropy in wide centrality bins," arXiv:1212.3650 [nucl-ex].

[6] V. Bairathi, M. R. Haque and B. Mohanty, "Selecting specific initial configurations using spectator neutrons in U+U collisions," Phys. Rev. C 91, no. 5, 054903 (2015) doi:10.1103/PhysRevC.91.054903 [arXiv:1504.04719 [nuclex]].

[7] M. R. Lomnitz [STAR Collaboration], "Measurement of Dmeson azimuthal anisotropy in $\mathrm{Au}+\mathrm{Au} 200 \mathrm{GeV}$ collisions at RHIC," arXiv:1601.00743 [hep-ex].

[8] M. Nasim and B. Mohanty, "Systematic study of the elliptic flow parameter using a transport approach," Int. J. Mod. Phys. E 24, no. 04, 1550027 (2015) doi:10.1142/S0218301315500275 [arXiv:1402.6093 [nucl-ex]].

[9] N. Borghini and J. Y. Ollitrault, "Azimuthally sensitive correlations in nucleus-nucleus collisions," Phys. Rev. C 70, 064905 (2004) [nucl-th/0407041].

[10] C. Adler et al. [STAR Collaboration], "Elliptic flow from two and four particle correlations in $\mathrm{Au}+\mathrm{Au}$ Phys. Rev. C 66, 034904 (2002) [nucl-ex/0206001].

[11] M. Luzum and J. -Y. Ollitrault, "The event-plane method is obsolete,” arXiv:1209.2323 [nucl-ex]. 